\title{
Analysis and Comparison of Different Cooperative Cellular Networks
}

By

Imbaby I. Mahmoud

Engineering Dept, Nuclear Research Centre,

Atomic Energy Authority, Inshas, Egypt

\section{Sherief Hashima}

Engineering Dept, Nuclear Research Centre,

Atomic Energy Authority, Inshas, Egypt
Osama H. Elgazzar

Engineering Dept, Nuclear Research Centre, Atomic Energy Authority, Inshas, Egypt

\section{H. A. Konber}

Electrical Engineering Dept, Faculty of Engineering, Al Azhar University, Egypt 


\section{Analysis and Comparison \\ of Different Cooperative Cellular Networks}

Imbaby I. Mahmoud

Sherief Hashima
Osama H. Elgazzar

H. A. Konber

\section{Abstract}

This paper addresses Co-Channel Interference (CCI) problem in cellular networks. Therefore, network performance improvements through reducing $\mathrm{CCI}$ are evaluated using different four interference mitigation techniques. These techniques represent traditional sectored Fractional Frequency Reuse (FFR), sectored FFR do Beamforming (BF), Relay Assisted (R-A) sectored FFR and R-A sectored FFR do BF technique. Additionally, each one of the techniques which cooperative BF covers the horizontal, vertical and Three Dimensional (3D) BF techniques. Consequently, sectored FFR do BF contains three different scenarios. These scenarios indicate sectored FFR do horizontal BF, sectored FFR do vertical BF and sectored FFR do 3D BF. Furthermore, there are three different configurations of applying BF in R-A sectored FFR do BF. These configurations signify Relay Station (RS) do BF only, Base Station (BS) do BF only and both RS and BS do BF. Therefore, each configuration consists of three different scenarios according to the applied BF technique. Consequently, nine different scenarios are studied. A comparison of all scenarios in different techniques is introduced to enhance the network Quality of Service (QoS). Analytical treatments are conducted. As a result, closed-form expressions for worstcases Cell Edge User's (CEU's) Signal to Interference Ratio (SIR), Cell Centre User's (CCU's) SIR and inner radius are implemented. These expressions are used to compare different techniques using various performance evaluation metrics. The results show, that as unpredicted, the 
performance of R-A sectored FFR is close to that of R-A sectored FFR BF in low SIR threshold and high PL exponent ranges. Therefore, R-A sectored FFR is preferred in use from the network cost and power consumption perspectives. Furthermore, vertical BF shows performance close to $3 \mathrm{D} \mathrm{BF}$ in low Path-loss (PL) exponent range. Therefore, applying vertical BF is preferable with regard to the network budget and energy preserve. Moreover, it is observed, that configuration of $\mathrm{BS}$ do $\mathrm{BF}$ is superior to other configurations when horizontal or vertical BF technique is applied. Additionally, the configuration of both RS and BS do BF outperforms the other configurations when $3 \mathrm{D}$ BF is applied. However, as unexpected, it performs worse than them when the other BF techniques are applied. The work outcomes attain much higher CEU's SIR improvement. Therefore, the outage probability is decreased. Thus, network can cover more users. Accordingly, the total network cost is reduced. So, this treatment improves the network performance while keeping lower network cost.

Keywords: Communication Networks, Correction Factor, Relay Assisted Systems, Interference Mitigation Techniques, Cooperative Cellular Systems.

\section{Introduction}

Co-Channel Interference (CCI) problem restricts achieving high system performance in cellular networks [1]. Therefore, Fractional Frequency Reuse (FFR) technique is addressed to solve this problem. This technique divides each cell into inner and outer region. Furthermore, the inner region serves Cell Center Users (CCUs) and uses Frequency Reuse Factor (FRF) of one. Moreover, the outer region deploys FRF greater than one to cover Cell Edge Users (CEUs) [2]. Regrettably, this technique hasn't the power to fully remove CCI problem [3]. Thus, Relay Assisted (R-A) cellular networks are introduced as another solution to erase this problem [4]. These networks have received particular consideration due to its aptitude 
to increase the coverage area and signal strength [5]. Therefore, an expression for the secrecy outage probability for Amplify and Forward (AF) relay network over Nakagami-m fading channels is introduced [6]. In addition, the performance of Decode and Forward (DF) relaying over an independent, asymmetrical and generalized fading channels is analyzed [7]. Moreover, a comparison between AF and DF Relay Stations (RSs) is implemented [8]. Unfortunately, it is found, that in these networks there are many issues affecting the system reliability. The first difficult represents the interference that generated from RSs deployment [9]. Furthermore, the second problem denotes the Bandwidth (BW) efficiency reduction due to the increase in number of deployed RSs [10]. Additionally, the third issue signifies the increase in power consumption due to the big difference in power levels of transmitted and received signals [11]. Consequently, Beamforming (BF) technique has been used to solve these issues. This alternative solution enables the antenna to direct the signal to the desired direction which reduces the interference level [12]. Hence, the joint user BF and relay transmit strategies to deal with the self-interference signal at the Full Duplex (FD) RS is studied [13]. Additionally, relay BF design to minimize the maximum interference caused at the neighboring cells is carried out [14].

The objective of this paper is to introduce an effective solution for CCI issue in OFDMA cellular networks. Thus, four different interference mitigation techniques are analytically treated. These techniques represent traditional sectored FFR, sectored FFR cooperative with different BF techniques, R-A sectored FFR and R-A sectored FFR cooperative with different BF techniques. Additionally, each one of the techniques which uses BF considers the horizontal, vertical and Three Dimensional (3D) BF techniques. Consequently, sectored FFR cooperative with BF contains three different scenarios. These scenarios denote sectored FFR do horizontal BF, sectored FFR do vertical BF and sectored FFR do 3D BF. Furthermore, 
three different configurations of deploying BF are considered in R-A sectored FFR cooperative with different $\mathrm{BF}$ techniques. These configurations indicate RS do BF only, BS do BF only and both $\mathrm{RS}$ and $\mathrm{BS}$ do $\mathrm{BF}$. Therefore, three configurations of applying BF and three BF techniques form nine different scenarios in total. Also, frequency allocation scheme is assumed for each technique to reduce the interference level. These expressions are then used to draw a comparison among these techniques using various performance evaluation metrics. Accordingly, the results of this work contribute to improving network Quality of Service (QoS) while keeping lower network cost.

This paper is organized as follows: the basic assumptions and models description are introduced in Sect. 2. Section 3 focuses on system performance evaluation metrics. Section 4 introduces the results and discussion. Section 5 is dedicated to the conclusion of the work.

\section{Basic Assumptions and Models Description}

This section presents the analysis of different interference mitigation models. These models denote traditional sectored FFR technique, sectored FFR do BF technique, R-A sectored FFR technique and R-A sectored FFR do $\mathrm{BF}$ technique. Furthermore, this analytical treatment resulted in closed form expressions for CCU's SIR and CEU's SIR as shown in the next subsections. These expressions are then used to compare the performance of different models.

\subsection{Analysis of traditional sectored FFR technique}

Two tier sectored FFR system is considered as revealed in Fig. 1 (a). In this system there are 19 cells and each cell is divided into inner and outer regions. Moreover, the outer region is divided into three sectors. Consequently, the total BW is divided equally between the inner region and each sector in the outer region. Therefore, inner region 
deploys Frequency Band $(\mathrm{FB})\left(\mathrm{f}_{1}\right)$ to cover CCUs. Additionally, outer region uses FBs $\left(f_{2}, f_{3}\right.$ and $\left.f_{4}\right)$ to serve CEUs as shown in Fig. 1 (b).

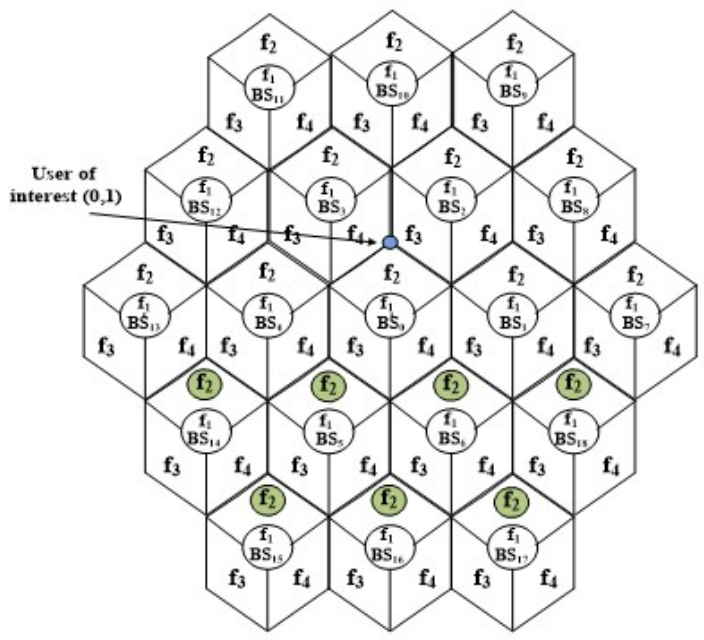

(a)

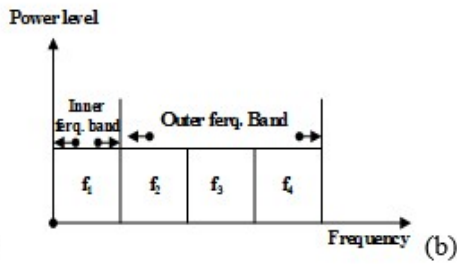

Fig. 1 Two tier sectored FFR system (a) system layout (b) frequency allocating scheme

Furthermore, with a view to control CCI in cellular networks, the received average SINR is used to classify different users as either CEUs or CCUs. Therefore, the user is classified as CEU when the received average SINR is below a given threshold. Moreover, when received SINR excessed that threshold, the user is categorized as CCU. Additionally, it is assumed, that the serving $\mathrm{BS}\left(\mathrm{BS}_{0}\right)$ located at the coordinate origin as revealed in Fig. 1 (a). Therefore, the SINR of a user $u$ $(\mathrm{X}, \mathrm{Y})$ located at a distance from $\mathrm{BS}_{0}$ is given by the following equation [15]

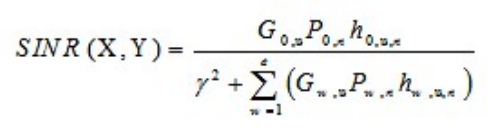

where $\mathrm{u}$ is the user index, $\mathrm{G}_{0, \mathrm{u}}$ represents the PL associated with the channel between user $\mathrm{u}$ and $\mathrm{BS}_{0}, \mathrm{G}_{\mathrm{w}, \mathrm{u}}$ denotes PL related to the channel between user $\mathrm{u}$ and interfering $\mathrm{BSs}, \mathrm{n}$ is the subcarrier index, $\mathrm{P}_{0, \mathrm{n}}$ refers to the 
transmitted power of $\mathrm{BS}_{0}$ on subcarrier $\mathrm{n}, \mathrm{w}$ is the interfering $\mathrm{BS}$ index, $\mathrm{e}$ denotes the number of interfering BSs (i.e. BSs that are using the same FB as $\mathrm{BS}_{0}$ ), $\mathrm{P}_{\mathrm{w}, \mathrm{n}}$ symbolizes the transmitted power of interfering BSs on subcarrier $\mathrm{n}, \mathrm{h}_{0, \mathrm{u}, \mathrm{n}}$ signifies the exponentially distributed channel Fast Fading (FF) power between user $\mathrm{u}$ and $\mathrm{BS}_{0}, \mathrm{~h}_{\mathrm{w}, \mathrm{u}, \mathrm{n}}$ characterizes the exponentially distributed channel FF power between user $u$ and interfering BSs and $\gamma^{2}$ is the noise power of the Additive White Gaussian Noise (AWGN) channel. Furthermore, the parameter $\mathrm{G}_{0, \mathrm{u}}$ is directly proportional to $\mathrm{r}^{-\eta}$ i.e. $\left(\mathrm{G}_{0, \mathrm{u}} \alpha \mathrm{r}^{-\eta}\right)$ where $\eta$ and $\mathrm{r}$ represent the PL exponent and distance between the BS and user $\mathrm{u}$, respectively. So, this relation can be formulated as $\mathrm{G}_{0, \mathrm{u}}=\mathrm{G}_{\mathrm{o}} \mathrm{r}^{-}$ $\eta$ where $G_{0}$ is the first proportional constant which can be defined as $\mathrm{G}_{\mathrm{o}}=\left(\mathrm{c} / 4 \pi \mathrm{f}_{\mathrm{o}}\right)^{2}$ where $\mathrm{f}_{\mathrm{o}}$ and $\mathrm{c}$ signify the center frequency of serving BS and speed of light, respectively. Moreover, the parameter $\mathrm{G}_{\mathrm{w}, \mathrm{u}}$ is defined as $G_{w, u}=G_{w} r_{w}^{-\eta}$ where $G_{w}$ and $r_{w}$ denote the second proportional constant and distance between the interfering BSs and user $\mathrm{u}$, respectively. Besides, the second proportional constant is defined as $G_{w}=\left(c / 4 \pi f_{w}\right)^{2}$ where $f_{w}$ is the center frequency of interfering BSs. The interfering sources are all neighboring $\mathrm{BSs}$ that use the same $\mathrm{FB}$ as $\mathrm{BS}_{0}$. Therefore, CCUs suffer from 18 interfering $\mathrm{BSs}\left(\mathrm{BS}_{1:} \mathrm{BS}_{18}\right)$ that use the same $\mathrm{FB}\left(\mathrm{f}_{1}\right)$ as $\mathrm{BS}_{0}$ in the inner region as depicted in Fig. 1 (a). Additionally, CEUs suffer from 7 interfering BSs which denote cells number $(5,6,14,15$, 16,17 and 18) that use $\mathrm{FB}\left(\mathrm{f}_{2}\right)$ as $\mathrm{BS}_{0}$ in the outer region. In addition, it is assumed, that channel fading powers are independent with unit mean, i.e. $\left(\mathrm{h}_{0, \mathrm{u}, \mathrm{n}}=1\right)$, and the main effect in cellular network comes from the interference than from noise. Thus, noise term is neglected in our analysis. Furthermore, user u can be considered either a CCU or CEU as he moves from the inner region to the cell edge. Thus, Eq. (1) is reformulated to calculate the CCU's SIR and CEU's SIR of the user u as follows [16] 


$$
\operatorname{SIR}(X, Y)=\frac{P_{0} G_{0} r^{-\pi}}{\left[Z \sum_{k=1}^{18} P_{k} G_{k} r_{k}^{-n}\right]+\left[Z \sum_{j S S=1}^{n} P_{j s S} G_{j S S} r_{j S S}-\pi\right]}
$$

where $\mathrm{P}_{0}$ denotes the transmitted power by $\mathrm{BS}_{0}, \mathrm{k}$ is the interfering BS index in the inner region due to reuse one frequency $\left(f_{1}\right), r_{k}$ represents the distance between CCU and the 18 interfering BSs that use FB $\left(\mathrm{f}_{1}\right), \mathrm{P}_{\mathrm{k}}$ is the transmitted power by the interfering BSs that affect $\mathrm{CCU}, \mathrm{G}_{\mathrm{k}}$ indicates the inner proportional constant which is defined as $\mathrm{G}_{\mathrm{k}}=\left(\mathrm{c} / 4 \pi \mathrm{f}_{\mathrm{k}}\right)^{2}$ where $\mathrm{f}_{\mathrm{k}}$ is the center frequency of interfering BSs which affect the $\mathrm{CCU}, \mathrm{jBS}$ is the interfering $\mathrm{BSs}$ index in the outer region due to reuse $\mathrm{FB}\left(\mathrm{f}_{2}\right), \mathrm{r}_{\mathrm{jBS}}$ characterizes the distance between CEU and 7 interfering BSs that use $\mathrm{FB}\left(\mathrm{f}_{2}\right), \mathrm{P}_{\mathrm{jBS}}$ is the transmitted power by the interfering $B S s, G_{j B S}$ signifies the outer proportional constant which is defined as $\mathrm{G}_{\mathrm{jBS}}=\left(\mathrm{c} / 4 \pi \mathrm{f}_{\mathrm{jBS}}\right)^{2}$ where $\mathrm{f}_{\mathrm{jBS}}$ is the center frequency of interfering BSs which affect $C E U, Z$ is the parameter that defines the user location if $\mathrm{CEU}(\mathrm{Z}=0)$ or $\mathrm{CCU}(\mathrm{Z}=1)$ and $\mathrm{Z}^{\prime}$ represents the complement of parameter $Z$. Additionally, it is assumed, that equal transmitted power is applied for all BSs and the interference of users is negligible. So, the general SIR expression for CCU can be written as follows

$$
S I R_{\text {CCU }}(X, Y)=\frac{G_{o} r^{-\eta}}{Z \sum_{k=1}^{18} G_{k} r_{k}^{-\eta}}
$$

where $\operatorname{SIR}_{\mathrm{CCU}}$ is the CCU's SIR. Also, the values of $\mathrm{G}_{\mathrm{o}}$ and $\mathrm{G}_{\mathrm{k}}$ are equal because all BSs in the inner region use the same frequency $\left(f_{1}\right)$. Moreover, the general equation of CEU's SIR under equal transmitted power assumption can be expressed by the following relation

$$
\operatorname{SIR}_{C E V}(X, Y)=\frac{G, r^{-n}}{Z \sum_{j S S-1}^{n} G_{\beta S S} r_{j S S^{-n}}}
$$


where SIR $_{\text {CEU }}$ is the CEU's SIR. In addition, the values of $\mathrm{G}_{\mathrm{o}}$ and $\mathrm{G}_{\mathrm{jBS}}$ are identical due to deploying the same $\mathrm{FB}\left(\mathrm{f}_{2}\right)$ by all BSs in the outer region. Thus, Eq. (4) can be reformulated as follows

$$
\operatorname{SIR}_{C E V}(X, Y)=\frac{r^{-n}}{\sum_{j=1}^{n} r_{P S}^{-n}}
$$

Additionally, it is assumed, that $(\mathrm{x}, \mathrm{y})$ are the normalized coordinates to the cell radius $\left(\mathrm{R}_{\text {Cell }}\right)$ i.e. $(\mathrm{x}, \mathrm{y})=\left(\mathrm{X} / \mathrm{R}_{\mathrm{Cell}}, \mathrm{Y} / \mathrm{R}_{\mathrm{Cell}}\right)$. So, the normalized distance will equal to. On the other hand, in the outer region, there are 7 interfering BSs that use the same $\mathrm{FB}\left(\mathrm{f}_{2}\right)$ as $\mathrm{BS}_{0}$ and affect CEU's SIR. Accordingly, Eq. (5) can be reformulated to represent the CEU's SIR expression in normalized coordinates as follows

$$
S I R_{S e c}(x, y)=\frac{\left(x^{2}+y^{2}\right)^{\sigma}}{I_{S e c}(x, y)}
$$

where $\mathrm{I}_{\mathrm{Sec}}$ is the interference factor due to neighboring BSs in sectored FFR system. Therefore, this parameter can be computed from the following relation

$$
I_{S s c}(x, y)=\sum_{j_{s S}-1}^{7}\left[\left(x-x_{j_{s s}}\right)^{2}+\left(y-y_{j_{s s}}\right)^{2}\right]^{\sigma}
$$

Furthermore, CEU located at the second corner with coordinates $(0$, 1) is assumed. Thus, by substituting the value of Eq. (7) into Eq. (6), the worst-case CEU's SIR can be calculated as follows

$$
S R R_{\text {Scc }}=\left[2\left(7^{\sigma}+13^{\sigma}+19^{\sigma}\right)+16^{\sigma}\right]^{-1}
$$

where SIR $_{\text {Sec }}$ denotes the worst-case CEU's SIR closed-form expression when sectored FFR technique is employed. 
Table 1 BSs coordinates and assigned frequencies for R-A sectored FFR system

\begin{tabular}{|c|c|c|c|c|c|c|}
\hline \multirow[t]{2}{*}{ BS } & \multirow[t]{2}{*}{$\mathbf{X}$} & \multirow[t]{2}{*}{$\mathbf{y}$} & \multirow[t]{2}{*}{$\begin{array}{l}\text { Inner Freq. } \\
\text { Bands }\end{array}$} & \multirow{2}{*}{$\begin{array}{c}\text { Sectored FFR } \\
\text { Frequency } \\
\text { Assignment } \\
\text { BSs Freq. Bands }\end{array}$} & \multicolumn{2}{|c|}{$\begin{array}{c}\text { R-A Sectored FFR Frequency } \\
\text { Assignment }\end{array}$} \\
\hline & & & & & RSs Freq. Bands & BSs Freq. Bands \\
\hline 0 & 0 & 0 & $\mathrm{f}_{1}$ & $\mathrm{f}_{2}, \mathrm{f}_{3}$ and $\mathrm{f}_{4}$ & $\mathrm{f}_{2-2}, \mathrm{f}_{3-2}$ and $\mathrm{f}_{4-2}$ & $f_{2-1}, f_{3-1}$ and $f_{4^{-1}}$ \\
\hline 1 & $\sqrt{3}$ & 0 & $\mathrm{f}_{1}$ & $\mathrm{f}_{2}, \mathrm{f}_{3}$ and $\mathrm{f}_{4}$ & $\mathrm{f}_{2-2}, \mathrm{f}_{3-2}$ and $\mathrm{f}_{4-2}$ & $f_{2-1}, f_{3-1}$ and $f_{4^{-1}}$ \\
\hline 2 & $\sqrt{3} / 2$ & $3 / 2$ & $\mathrm{f}_{1}$ & $\mathrm{f}_{2}, \mathrm{f}_{3}$ and $\mathrm{f}_{4}$ & $\mathrm{f}_{2-2}, \mathrm{f}_{3-2}$ and $\mathrm{f}_{4-2}$ & $\mathrm{f}_{2-1}, \mathrm{f}_{3-1}$ and $\mathrm{f}_{4^{-}-1}$ \\
\hline 3 & $-\sqrt{ } 3 / 2$ & $3 / 2$ & $\mathrm{f}_{1}$ & $\mathrm{f}_{2}, \mathrm{f}_{3}$ and $\mathrm{f}_{4}$ & $\mathrm{f}_{2-2}, \mathrm{f}_{3-2}$ and $\mathrm{f}_{4-2}$ & $\mathrm{f}_{2-1}, \mathrm{f}_{3-1}$ and $\mathrm{f}_{4^{-}-1}$ \\
\hline 4 & $-\sqrt{3}$ & 0 & $\mathrm{f}_{1}$ & $\mathrm{f}_{2}, \mathrm{f}_{3}$ and $\mathrm{f}_{4}$ & $\mathrm{f}_{2-2}, \mathrm{f}_{3-2}$ and $\mathrm{f}_{4-2}$ & $\mathrm{f}_{2-1}, \mathrm{f}_{3-1}$ and $\mathrm{f}_{4^{-}-1}$ \\
\hline 5 & $-\sqrt{ } 3 / 2$ & $3 / 2$ & $\mathrm{f}_{1}$ & $\mathrm{f}_{2}, \mathrm{f}_{3}$ and $\mathrm{f}_{4}$ & $\mathrm{f}_{2-2}, \mathrm{f}_{3-2}$ and $\mathrm{f}_{4-2}$ & $f_{2-1}, f_{3-1}$ and $f_{4^{-1}}$ \\
\hline 6 & $\sqrt{3} / 2$ & $\begin{array}{c}- \\
3 / 2\end{array}$ & $\mathrm{f}_{1}$ & $\mathrm{f}_{2}, \mathrm{f}_{3}$ and $\mathrm{f}_{4}$ & $\mathrm{f}_{2-2}, \mathrm{f}_{3-2}$ and $\mathrm{f}_{4-2}$ & $\mathrm{f}_{2-1}, \mathrm{f}_{3-1}$ and $\mathrm{f}_{4^{-1}}$ \\
\hline 7 & $2 \sqrt{ } 3$ & 0 & $f_{1}$ & $\mathrm{f}_{2}, \mathrm{f}_{3}$ and $\mathrm{f}_{4}$ & $\mathrm{f}_{2-2}, \mathrm{f}_{3-2}$ and $\mathrm{f}_{4-2}$ & $\mathrm{f}_{2-1}, \mathrm{f}_{3-1}$ and $\mathrm{f}_{4^{-}-1}$ \\
\hline 8 & $3 \sqrt{3} / 2$ & $3 / 2$ & $\mathrm{f}_{1}$ & $\mathrm{f}_{2}, \mathrm{f}_{3}$ and $\mathrm{f}_{4}$ & $\mathrm{f}_{2-2}, \mathrm{f}_{3-2}$ and $\mathrm{f}_{4-2}$ & $\mathrm{f}_{2-1}, \mathrm{f}_{3-1}$ and $\mathrm{f}_{4^{-1}}$ \\
\hline 9 & $\sqrt{3}$ & 3 & $\mathrm{f}_{1}$ & $\mathrm{f}_{2}, \mathrm{f}_{3}$ and $\mathrm{f}_{4}$ & $f_{2-2}, f_{3-2}$ and $f_{4-2}$ & $f_{2-1}, f_{3-1}$ and $f_{4^{-1}}$ \\
\hline 10 & 0 & 3 & $\mathrm{f}_{1}$ & $\mathrm{f}_{2}, \mathrm{f}_{3}$ and $\mathrm{f}_{4}$ & $\mathrm{f}_{2-2}, \mathrm{f}_{3-2}$ and $\mathrm{f}_{4-2}$ & $\mathrm{f}_{2-1}, \mathrm{f}_{3-1}$ and $\mathrm{f}_{4-1}$ \\
\hline 11 & $-\sqrt{3}$ & 3 & $\mathrm{f}_{1}$ & $\mathrm{f}_{2}, \mathrm{f}_{3}$ and $\mathrm{f}_{4}$ & $\mathrm{f}_{2-2}, \mathrm{f}_{3-2}$ and $\mathrm{f}_{4-2}$ & $\mathrm{f}_{2-1}, \mathrm{f}_{3-1}$ and $\mathrm{f}_{4^{-}-1}$ \\
\hline 12 & $-3 \sqrt{ } 3 / 2$ & $3 / 2$ & $\mathrm{f}_{1}$ & $\mathrm{f}_{2}, \mathrm{f}_{3}$ and $\mathrm{f}_{4}$ & $\mathrm{f}_{2-2}, \mathrm{f}_{3-2}$ and $\mathrm{f}_{4-2}$ & $\mathrm{f}_{2-1}, \mathrm{f}_{3-1}$ and $\mathrm{f}_{4^{-1}}$ \\
\hline 13 & $-2 \sqrt{3}$ & 0 & $\mathrm{f}_{1}$ & $\mathrm{f}_{2}, \mathrm{f}_{3}$ and $\mathrm{f}_{4}$ & $\mathrm{f}_{2-2}, \mathrm{f}_{3-2}$ and $\mathrm{f}_{4-2}$ & $\mathrm{f}_{2-1}, \mathrm{f}_{3-1}$ and $\mathrm{f}_{4^{-1}}$ \\
\hline 14 & $-3 \sqrt{ } 3 / 2$ & $3 / 2$ & $\mathrm{f}_{1}$ & $\mathrm{f}_{2}, \mathrm{f}_{3}$ and $\mathrm{f}_{4}$ & $\mathrm{f}_{2-2}, \mathrm{f}_{3-2}$ and $\mathrm{f}_{4-2}$ & $\mathrm{f}_{2-1}, \mathrm{f}_{3-1}$ and $\mathrm{f}_{4^{-1}}$ \\
\hline 15 & $-\sqrt{3}$ & -3 & $\mathrm{f}_{1}$ & $\mathrm{f}_{2}, \mathrm{f}_{3}$ and $\mathrm{f}_{4}$ & $\mathrm{f}_{2-2}, \mathrm{f}_{3-2}$ and $\mathrm{f}_{4-2}$ & $\mathrm{f}_{2-1}, \mathrm{f}_{3-1}$ and $\mathrm{f}_{4^{-} 1}$ \\
\hline 16 & 0 & -3 & $\mathrm{f}_{1}$ & $\mathrm{f}_{2}, \mathrm{f}_{3}$ and $\mathrm{f}_{4}$ & $\mathrm{f}_{2-2}, \mathrm{f}_{3-2}$ and $\mathrm{f}_{4-2}$ & $\mathrm{f}_{2-1}, \mathrm{f}_{3-1}$ and $\mathrm{f}_{4^{-1}}$ \\
\hline 17 & $\sqrt{3}$ & -3 & $f_{1}$ & $\mathrm{f}_{2}, \mathrm{f}_{3}$ and $\mathrm{f}_{4}$ & $\mathrm{f}_{2-2}, \mathrm{f}_{3-2}$ and $\mathrm{f}_{4-2}$ & $\mathrm{f}_{2-1}, \mathrm{f}_{3-1}$ and $\mathrm{f}_{4^{-}}$ \\
\hline 18 & $3 \sqrt{ } 3 / 2$ & $3 / 2$ & $\mathrm{f}_{1}$ & $\mathrm{f}_{2}, \mathrm{f}_{3}$ and $\mathrm{f}_{4}$ & $f_{2-2}, f_{3-2}$ and $f_{4-2}$ & $\mathrm{f}_{2-1}, \mathrm{f}_{3-1}$ and $\mathrm{f}_{4^{-1}}$ \\
\hline
\end{tabular}




\subsection{Analysis of sectored FFR do BF technique}

Although the interfering signals coming from neighboring cells are reduced by using the sectored FFR technique, it can't be removed completely. To overcome this problem, BF techniques with smart antenna systems have been utilized for further network performance development. These BF techniques denote the horizontal, vertical and 3D BF techniques. The basic feature of the horizontal BF technique is that, BS can simultaneously provide service to several Mobile Stations (MSs) located in different horizontal directions by choosing beams with different azimuths. Consequently, the desired signal power increases and the CCI from adjacent cells decreases. Furthermore, the horizontal antenna gain $\mathrm{A}(\theta)$ for each sector antenna due to horizontal BF employment is expressed as follows [17]

$$
A(\theta)=-\min \left(12\left[\frac{\theta}{\theta_{3 a B}}\right], G_{m}\right)
$$

where $A(\theta)$ indicates the antenna gain at an angle $\theta$ in decibels $(\mathrm{dB})$, $\theta$ represents the angle between direction of interest and boresight of antenna in horizontal dimension, $\theta_{3 \mathrm{~dB}}$ is the $3 \mathrm{~dB}$ beamwidth of horizontal beam and $\mathrm{G}_{\mathrm{m}}$ front to back attenuation. However, it is found, that all MSs that are served by beams with the same down-tilt angle can't be distinguished at the same time [18] as depicted in Fig. 2. Therefore, deploying the horizontal BF technique in this case is not the best choice to cover many CEUs simultaneously. Consequently, the vertical BF technique is used to perform this target. 


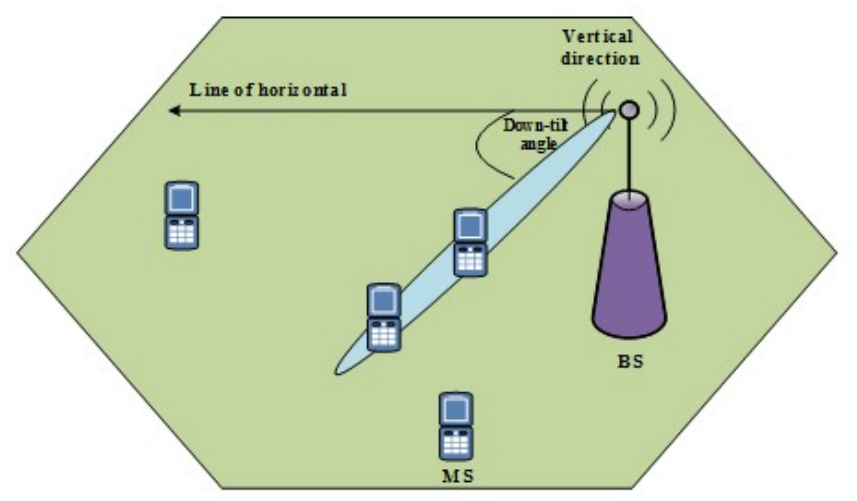

Fig. 2 Drawback of horizontal BF technique

The vertical antenna gain $\mathrm{A}(\phi)$ is calculated from the following relation [18]

$$
A(\phi)=-\min \left(12\left[\frac{\phi-\phi_{\text {iti }}}{\phi_{\text {SaB }}}\right], S L G_{v}\right)
$$

where $\mathrm{A}(\phi)$ represents the antenna gain at an angle $\phi$ in $\mathrm{dB}, \phi$ symbolizes the angle between the direction of interest and the boresight of the antenna in vertical dimension, $\phi_{3 \mathrm{~dB}}$ characterizes the angle of which the antenna gain is $3 \mathrm{~dB}$ lower than the antenna gain in the main-beam direction of the vertical beam, $\phi_{\text {tilt }}$ denotes the down-tilt angle in the transmitter and $\mathrm{SLG}_{\mathrm{v}}$ is the maximum attenuation of the side-lobe. Additionally, the parameters $\mathrm{G}_{\mathrm{m}}$ and $\mathrm{SLG}_{\mathrm{v}}$ are set to be $20 \mathrm{~dB}$. Also, the reference values of these parameters are taken from [17] and [18]. The BF concept for both horizontal and vertical BF techniques is shown in Fig. 3.

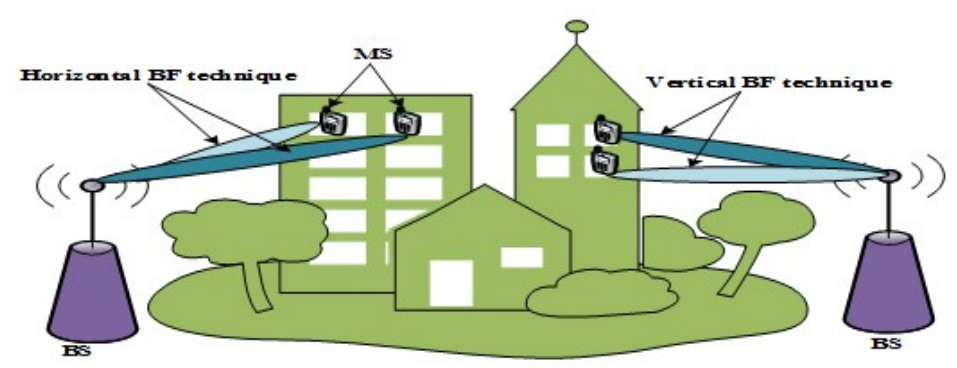


Fig. 3 Concept illustration of horizontal and vertical BF techniques

On the other hand, the rapid growth of high rise buildings in modern cities results in a growing range of floors in which users may stay on. Thus, 3D BF technique is used where it can improve the transmission efficiency greatly over the Two-Dimensional (2D) BF techniques such as (horizontal and vertical BF techniques) [18] as shown in Fig. 4.

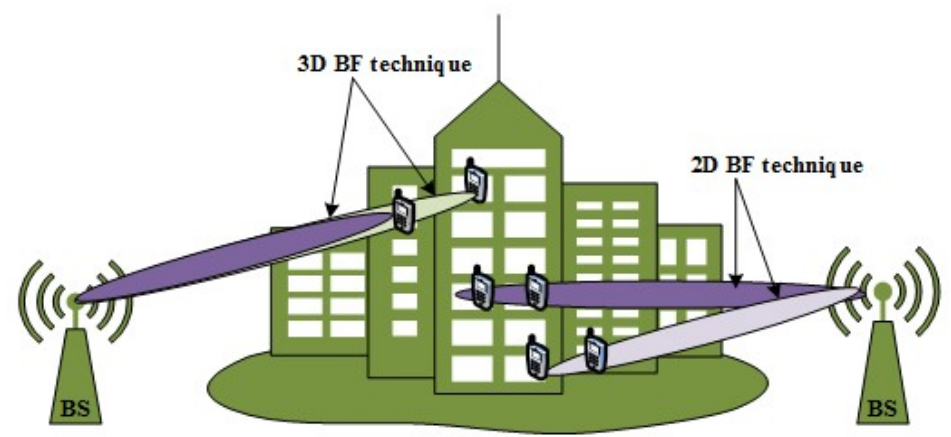

Fig. 4 Difference illustration between 2D BF and 3D BF technique

The 3D antenna gain is the sum of the horizontal and elevation patterns antenna gain. Therefore, generation of 3D pattern from two perpendicular cross-sections azimuth and elevation patterns is given by the following formula [18]

$$
A(\theta, \phi)=-\min \left(-[A(\theta)+A(\phi)], G_{m}\right)
$$

where $\mathrm{A}(\theta, \phi)$ is the $3 \mathrm{D}$ antenna gain at angels $\theta$ and $\phi$ in decibels (dB). Therefore, CEU's SIR of the considered model is calculated by applying these BF techniques with BS to reduce CCI effect. Thus, Eq. (8) can be reformulated to calculate CEU's SIR expression as follows

$$
\operatorname{SIR}_{B r g s}(x, y)=\frac{A_{s s}(\theta, \phi)\left(x^{2}+y^{2}\right)^{\sigma}}{\sum_{j s s-1}^{7} A_{j s s}\left(\theta_{I}, \phi_{I}\right)\left[\left(x-x_{j s s}\right)^{2}+\left(y-y_{j s s}\right)^{2}\right]^{c}}
$$


where SIR $_{\mathrm{BFBS}}$ denotes the CEU's SIR after applying BF technique with $\mathrm{BS}, \mathrm{A}_{\mathrm{BS}}(\theta, \phi)$ represents the gain in $(\theta, \phi)$ directions of serving $\mathrm{BS}$ in the outer region and $\mathrm{A}_{\mathrm{jBS}}\left(\theta_{\mathrm{I}}, \phi_{\mathrm{I}}\right)$ characterizes the gain in $\left(\theta_{\mathrm{I}}\right.$ and $\left.\phi_{\mathrm{I}}\right)$ directions of the interfering BS in the outer region. In addition, the parameters $A(\theta, \phi)$ and $A_{j B S}\left(\theta_{I}, \phi_{I}\right)$ can be calculated from Eq. (9) when horizontal $\mathrm{BF}$ is applied. Also, these parameters can be obtained from Eqs. (10) and (11) when vertical and 3D BF are respectively deployed. Consequently, three different scenarios in this model are considered. These scenarios represent sectored FFR do horizontal BF, sectored FFR do vertical $\mathrm{BF}$ and sectored FFR do 3D BF. Additionally, the exact values of $A_{B S}(\theta, \phi)$ and $A_{j B S}\left(\theta_{I}, \phi_{I}\right)$ are conducted for different $B F$ techniques. Hence, a formula describing the CEU's SIR of sectored FFR do horizontal BF scenario is implemented and simplified as follows

$$
S I R_{H B S}=W_{10}\left(\left[7^{\sigma} W_{3} / \mathrm{W}_{2}\right]+\left[13^{\sigma} W_{6} / \mathrm{T}_{1}\right]+\left[16^{\sigma} T_{2} / \mathrm{W}_{2}\right]+\left[19^{\sigma} T_{3} / \mathrm{W}_{8}\right]\right)^{-1}
$$

where $\operatorname{SIR}_{\mathrm{HBS}}$ indicates the attained CEU's SIR from the scenario of sectored FFR do horizontal BF. Moreover, $\mathrm{W}_{2}, \mathrm{~W}_{3}, \mathrm{~W}_{6}, \mathrm{~W}_{8}, \mathrm{~W}_{10}, \mathrm{~T}_{1}$, $\mathrm{T}_{2}$ and $\mathrm{T}_{3}$ denote constant values shown in Table 2. Furthermore, for the scenario of sectored FFR do vertical BF, the CEU's SIR equation is calculated and simplified as follows

$$
S R_{V B S}=O_{8}\left(\left[7^{\sigma} O_{3} / \mathrm{O}_{4}\right]+\left[13^{\sigma} \mathrm{O}_{6} / \mathrm{X}_{1}\right]+\left[16^{\sigma} \mathrm{X}_{2} / \mathrm{X}_{3}\right]+\left[19^{\sigma} X_{4} / \mathrm{O}_{2}\right]\right)^{-1}
$$

where SIR ${ }_{V B S}$ symbolizes the achieved CEU's SIR from the scenario of sectored FFR do vertical BF. Furthermore, $\mathrm{O}_{2}, \mathrm{O}_{3}, \mathrm{O}_{4}, \mathrm{O}_{6}, \mathrm{O}_{8}, \mathrm{X}_{1}, \mathrm{X}_{2}$, $\mathrm{X}_{3}$ and $\mathrm{X}_{4}$ represent constant values displayed in Table 2. Moreover, the effect of applying 3D BF technique with sectored FFR is computed and reformulated as follows

$$
S I R_{3 D E S}=M_{17}\left(\left[7^{\sigma} M_{4} / M_{2}\right]+\left[13^{\sigma} M_{6} / M_{3}\right]+\left[16^{\sigma} M_{13} / M_{2}\right]+\left[19^{\sigma} M_{7} / M_{3}\right]\right)^{-1}
$$


where $\operatorname{SIR}_{3 \mathrm{DBS}}$ represents the reached CEU's SIR from the scenario of sectored FFR do 3D BF. Also, $\mathrm{M}_{2}, \mathrm{M}_{3}, \mathrm{M}_{4}, \mathrm{M}_{6}, \mathrm{M}_{7}, \mathrm{M}_{13}$ and $\mathrm{M}_{17}$ symbolize constant values shown in Table 2 .

Table 2 Parameters' values of sectored FFR do BF

\begin{tabular}{||c|c|c||}
\hline $\begin{array}{c}\text { Sectored FFR do hor. } \\
\text { BF }\end{array}$ & $\begin{array}{c}\text { Sectored FFR do } \\
\text { vertical BF }\end{array}$ & Sectored FFR do 3D BF \\
\hline \hline $\mathrm{W}_{2}=2.251799813 \times 10^{15}$ & $\mathrm{O}_{2}=4.503599627 \times 10^{15}$ & $\mathrm{M}_{2}=1.152921 \times 10^{15}$ \\
\hline $\mathrm{W}_{3}=1.37339601 \times 10^{15}$ & $\mathrm{O}_{3}=1.437387172 \times 10^{15}$ & $\mathrm{M}_{3}=5.7646075 \times 10^{15}$ \\
\hline $\mathrm{W}_{6}=3.022994811 \times 10^{15}$ & $\mathrm{O}_{4}=1.125899906 \times 10^{15}$ & $\mathrm{M}_{4}=1.68537439 \times 10^{15}$ \\
\hline $\mathrm{W}_{8}=2.81474976 \times 10^{14}$ & $\mathrm{O}_{6}=2.875753244 \times 10^{15}$ & $\mathrm{M}_{6}=7.552021340 \times 10^{15}$ \\
\hline $\mathrm{W}_{10}=2.0911$ & $\mathrm{O}_{8}=0.8589$ & $\mathrm{M}_{7}=8.117761096 \times 10^{15}$ \\
\hline $\mathrm{T}_{1}=1.125899906 \times 10^{15}$ & $\mathrm{X}_{1}=2.251799813 \times 10^{15}$ & $\mathrm{M}_{13}=7.77806801 \times 10^{15}$ \\
\hline $\mathrm{T}_{2}=2.013177238 \times 10^{15}$ & $\mathrm{X}_{2}=1.79642069 \times 10^{14}$ & $\mathrm{M}_{17}=0.9175$ \\
\hline $\mathrm{T}_{3}=1.465097859 \times 10^{15}$ & $\mathrm{X}_{3}=2.814749763 \times 10^{15}$ & \\
\hline & $\mathrm{X}_{4}=5.750823694 \times 10^{15}$ & \\
\hline
\end{tabular}

\subsection{Analysis of R-A sectored FFR technique}

Two tier R-A sectored FFR system is assumed as shown in Fig. 5 (a). This system is the same as sectored FFR but with employing relaying technique to improve the link performance and reliability. Thus, in this system, each cell has a BS and a certain number of RSs arranged in a regular 
pattern at distance equal to $R_{d}\left(R_{d}=0.75\right.$ of $\left.R_{\text {Cell }}\right)$ from the $B S$ as shown in Fig. 5 (b). Consequently, each FB in outer region is further portioned in two different sets of sub-bands to reduce the interference level between the RSs and BSs. The first set represents $\left(f_{2-1}, f_{3-1}\right.$ and $\left.f_{4-1}\right)$ sub-bands which are allocated to BSs. In addition, the second set denotes $\left(f_{2-2}, f_{3-2}\right.$ and $\left.f_{4-2}\right)$ subbands which are assigned to RSs as depicted in Fig. 5 (c). Furthermore, in this system, FD AF RSs are considered due to their simplicity and efficiency in utilizing spectrum resources. Accordingly, the communication is completed in two time slots. In first time slot, RS remains idle, while BS transmits the signal to both RS and MS through relay and direct link, respectively. In second time slot, RS forwards the previously received signal to the MS through access link after amplification process whereas the BS remains silent.

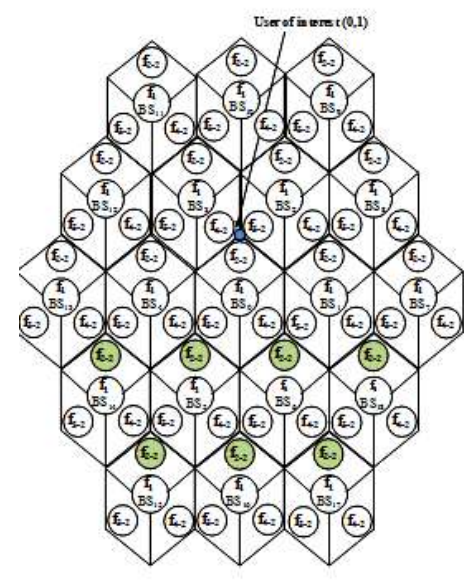

(a)
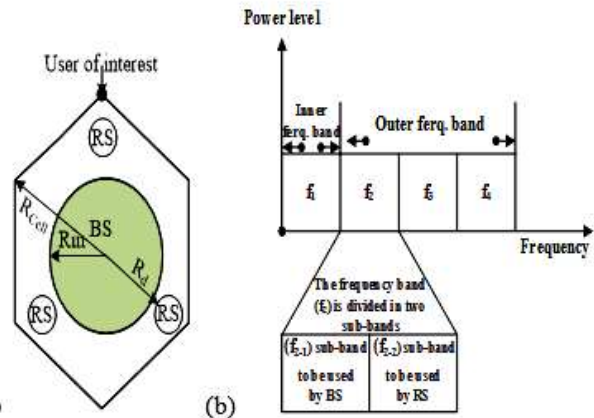

Fig. 5 Two-tier R-A sectored FFR system (a) system layout (b) cell layout (c) assigned frequency division

Consequently, MS combines these two time slots' SIRs by using Maximum Ratio Combined (MRC) technique as shown in Fig. 6. As a result, the CEU's SIR expression at MS is calculated as follows [19]

$$
M R C_{S R R}=1^{s t} T . S_{S I R}+2^{s c} T \cdot S_{S S R}
$$


where MRC $\mathrm{MIIR} 1^{\text {st }}$ T.S.SIR and $2^{\text {st }}$ T.S.SIR denote the MRC of the two time slots' SIRs, obtained CEU's SIR from the first time slot and achieved CEU's SIR from the second time slot, respectively. Thus, the analysis of two time slots is conducted in the following two subsections to calculate MRC closed form expression.

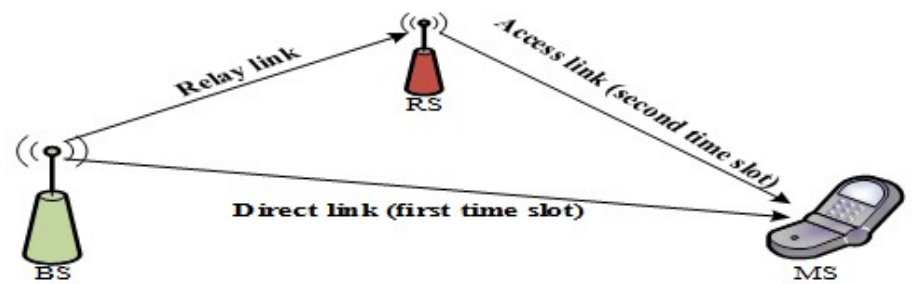

Fig. 6 Concept illustration of maximum ratio combining technique

\subsubsection{Analysis of first time slot}

In this time slot, BS transmits the signal to both RS and MS while RS keeps idle. Thus, the interfering sources are all neighboring BSs that use the same FB as the serving BS. Therefore, CCUs suffer from 18 interfering BSs resulted from reuse one frequency $\left(f_{1}\right)$ in the inner region as depicted in Fig. 5 (a). Furthermore, CEU suffers from 7 interfering cells that use the subband frequency $\left(f_{2-1}\right)$ in cells number $(5,6,14,15,16,17$, and 18). Therefore, the analysis of this time slot is identical with the sectored FFR analysis because in this time slot the communication is done completely by the BS. So, CEU's SIR closed form expression can be evaluated from Eq. (8).

\subsubsection{Analysis of second time slot}

In this time slot, each RS forwards its amplified signal to MS while BSs are still. Therefore, the worst case CEU's SIR of user u located at cell edge in the second time slot is estimated by the following formula 


$$
\operatorname{SIR}_{\text {Rse }}(x, y)=\frac{\left(x^{2}+(y-0.75)^{2}\right)^{\sigma}}{I_{\text {Rsex }}(x, y)}
$$

where $\mathrm{I}_{\mathrm{Rsec}}$ is the interference factor due to CCI from all RSs in neighboring cells that use the same sub-band frequency as the serving RS. It is considered, that user of interest is served by RS which deploys sub-band frequency $\left(f_{2-2}\right)$ in the central cell. So, there are 7 interfering RSs in cells number $(5,6,14,15,16,17$ and 18) that use the same sub-band frequency $\left(\mathrm{f}_{2-2}\right)$ as shown in Fig. 5 (a). The coordinates of these 7 interfering RSs are shown in Table 3. Consequently, the parameter $I_{\text {Rsec }}$ is evaluated from the following formula

$$
I_{\mathrm{Rsec}}(x, y)=\sum_{j_{R}=1}^{j_{R}-7}\left[\left(x+x_{j_{R}}\right)^{2}+\left(y+y_{j_{R}}\right)^{2}\right]^{\sigma}
$$

where $\mathrm{j}_{\mathrm{R}}$ denotes the group of interfering RSs that use the same subband frequency $\left(\mathrm{f}_{2-2}\right)$ as the serving RS. By substituting the value of Eq. (18) into Eq. (17), the worst case SIR of CEU with coordinates $(0,1)$ is expressed as follows

$$
S_{R \operatorname{Rec}}=(1 / 16)^{\sigma}\left[2\left((61 / 16)^{\sigma}+(157 / 16)^{\sigma}+(217 / 16)^{\sigma}\right)+(169 / 16)^{\sigma}\right]^{-1}
$$

where SIR $_{\text {Rsec }}$ signifies CEU's SIR of the second time slot for R-A sectored FFR network. 
Table 3 Interfering RSs 'Coordinates for R-A sectored FFR network

\begin{tabular}{|c|c|c||}
\hline $\begin{array}{c}\text { Interfering RS coordinates } \\
\text { in cell no }\end{array}$ & $\mathbf{X}$ & $\mathbf{Y}$ \\
\hline \hline 0 & $r / r V_{-}$ & $\varepsilon / r_{-}$ \\
\hline$\tau$ & $r / r V^{-}$ & $\varepsilon / r_{-}$ \\
\hline $1 \varepsilon$ & $r / r \sqrt{ } r_{-}$ & $\varepsilon / r_{-}$ \\
\hline 10 & $r V_{-}$ & $\varepsilon / q_{-}$ \\
\hline $1 \tau$ & $\cdot$ & $\varepsilon / q_{-}$ \\
\hline $1 V$ & $r \sqrt{ }$ & $\varepsilon / q_{-}$ \\
\hline 11 & $r / r \sqrt{ }$ & $\varepsilon / r_{-}$ \\
\hline
\end{tabular}

Accordingly, by substituting the values of Eq. (8) and Eq. (19) into Eq. (16), the worst case MRC closed form formula for R-A sectored FFR can be computed.

\subsection{Analysis of R-A sectored FFR do BF technique}

Although RSs deployment attains several benefits to the communication systems, it causes additional interference. Thus, BF techniques have been addressed to reduce interference related to RSs employment. The network performance improvements through reducing $\mathrm{CCI}$ are evaluated using three different configurations of applying BF. These configurations represent RS do BF only, BS do BF only and both RS and BS do BF. Also, in this technique, the considered BF techniques denote the horizontal, vertical and 3D BF technique. Therefore, each configuration consists of three different scenarios. Accordingly, nine different scenarios are studied. Therefore, MRC of the considered technique is calculated by 
applying the BF techniques with the two time slots as shown in the following two subsections.

\subsubsection{Analysis of first time slot}

In this time slot, as mentioned above, RS remains silent and BS transmits the signal to MS and RS. Therefore, BF techniques are applied with BS to reduce CCI effect. Thus, three different scenarios in this time slot are considered. These scenarios represent BS do horizontal BF, BS do vertical $\mathrm{BF}$ and $\mathrm{BS}$ do $3 \mathrm{D} \mathrm{BF}$. Here, the interfering sources are all neighboring BSs that use the same FB as the serving BS. Thus, CCUs suffer from 18 interfering BSs resulted from reuse one frequency $\left(f_{1}\right)$ in the inner region as depicted in Fig. 5 (a). Furthermore, CEU suffers from 7 interfering BSs that use the sub-band frequency $\left(f_{2-1}\right)$ in cells number $(5,6,14,15,16,17$ and 18). Accordingly, the analysis of this time slot is identical with sectored FFR do BF technique analysis because in this time slot the communication is totally completed through the BS. Consequently, Eqs. (13), (14) and (15) can be used to represent CEU's SIR closed form expressions when horizontal, vertical and 3D BF techniques are applied, respectively. Thus, by substituting the values of Eqs. (13) and (19) into Eq. (16), the MRC expression for the BS do horizontal BF scenario can be calculated. Moreover, by substituting the value of Eqs. (14) and (19) into Eq. (16), the MRC closed form formula for the scenario of BS do vertical BF can be computed

\subsubsection{Analysis of second time slot}

In this time slot, as discussed above, RS amplifies the previously received signal and forwards it to the MS where BS stills idle. So, BF techniques are applied with RS only. Thus, CEU's SIR formula of this system is calculated by considering BF effect with Eq. (17) as follows 


$$
\operatorname{SIR}_{\mathrm{RBF}}(x, y)=\frac{A_{R S}(\theta, \phi)\left(x^{2}+(y-0.75)^{2}\right)^{\sigma}}{\sum_{j_{R S}-1}^{7} A_{j_{2 S}}\left(\theta_{I}, \phi_{I}\right)\left[\left(x-x_{j_{R S}}\right)^{2}+\left(y-y_{j_{R S}}\right)^{2}\right]^{\sigma}}
$$

where SIR $_{\mathrm{RBF}}$ indicates the CEU's SIR of second time slot after applying $\mathrm{BF}$ technique with $\mathrm{RS}, \mathrm{A}_{\mathrm{RS}}\left(\theta_{\mathrm{I}}, \phi_{\mathrm{I}}\right)$ represents the gain in $(\theta$ and $\phi)$ directions of serving RS and $\mathrm{A}_{\mathrm{jRS}}\left(\theta_{\mathrm{I}}, \phi_{\mathrm{I}}\right)$ symbolizes gain in $\left(\theta_{\mathrm{I}}\right.$ and $\left.\phi_{\mathrm{I}}\right)$ directions of the interfering RS. Accordingly, in this time slot, there are another three different scenarios. These scenarios signify RS do horizontal BF, RS do vertical BF and RS do 3D BF. For the scenario of RS do horizontal BF, the CEU's SIR equation is evaluated and simplified as follows

$$
\operatorname{SIR}_{H R}=4^{\sigma} W_{10}\left(\left[19^{\sigma} W_{3} / W_{2}\right]+\left[43^{\sigma} W_{4} / \mathrm{W}_{5}\right]+\left[49^{\sigma} W_{6} / \mathrm{W}_{2}\right]+\left[61^{\sigma} W_{7} / \mathrm{W}_{8}\right]\right)^{-1}
$$

where SIR $_{\text {HR }}$ denotes the attained CEU's SIR from the scenario of RS do horizontal BF technique. Additionally, $\mathrm{W}_{5}$ and $\mathrm{W}_{7}$ are constant values presented in Table 4. Accordingly, by substituting the value of Eq. (8) and Eq. (21) in Eq. (16), the worst-case MRC closed-form formula for this scenario can be formulated. Additionally, the effect of deploying vertical BF technique with RS is computed and simplified as follows

$$
S_{I R}=4^{\sigma} O_{8}\left(\left[19^{\sigma} O_{3} / O_{4}\right]+\left[43^{\sigma} O_{5} / O_{2}\right]+\left[49^{\sigma} O_{6} / O_{2}\right]+\left[61^{\sigma} O_{7} / O_{2}\right]\right)^{-1}
$$

where SIR $_{V R}$ symbolizes the achieved CEU's SIR from the scenario of RS do vertical BF. Moreover, $\mathrm{O}_{5}$ and $\mathrm{O}_{7}$ denote constant values displayed in Table 4. Therefore, by substituting the value of Eq. (8) and Eq. (22) in Eq. (16), the worst-case MRC closed-form formula for this scenario can be evaluated. Furthermore, for the scenario of RS do 3D BF, the CEU's SIR equation is evaluated as follows

$$
S I R_{3 D R}=4^{\sigma} M_{17}\left(\left[19^{\sigma} M_{4} / M_{2}\right]+\left[43^{\sigma} M_{5} / M_{8}\right]+\left[49^{\sigma} M_{6} / M_{2}\right]+\left[61^{\sigma} M_{7} / M_{3}\right]\right)^{-1}
$$

where $\mathrm{SIR}_{3 \mathrm{DR}}$ indicates the obtained CEU's SIR from the scenario of RS do 3D BF. Furthermore, $\mathrm{M}_{5}$ and $\mathrm{M}_{8}$ denote constant values demonstrated 
in Table 4. Consequently, by substituting the value of Eq. (8) and Eq. (23) in Eq. (16), the worst-case MRC closed-form expression for this scenario can be calculated

Table 4 Parameters' values of RS do BF configuration

\begin{tabular}{||l|r|r||}
\hline \hline RS do horizontal BF & RS do vertical BF & RS do 3D BF \\
\hline $\mathrm{W}_{5}=5.6294995 \times 10^{14}$ & $\mathrm{O}_{5}=5.750026357 \times 10^{15}$ & $\mathrm{M}_{5}=1.916261 \times 10^{15}$ \\
\hline $\mathrm{W}_{7}=1.4650978 \times 10^{15}$ & $\mathrm{O}_{7}=5.750823694 \times 10^{15}$ & $\mathrm{M}_{8}=1.4411518 \times 10^{17}$ \\
\hline
\end{tabular}

Moreover, if both BS and RS apply different BF techniques, there will be additional three different scenarios. These scenarios represent both RS and BS do horizontal BF, both RS and BS do vertical BF and both RS and $\mathrm{BS}$ do $3 \mathrm{D} \mathrm{BF}$.

\section{System Performance Evaluation Metrics}

In this section, performance evaluation metrics are used to compare different configurations and scenarios. These metrics represent the channel capacity and Spectral Efficiency (SE).

\section{Different cellular networks spectral efficiency estimation}

SE refers to the rate of information which can be transmitted over a certain BW. So, it is a measure of how efficiently a restricted frequency spectrum is used. Accordingly, the SE is evaluated as follows [16]

$$
\left.S E=1 / N^{\log _{2}(1+C E U} s, S I R\right)
$$

where $\mathrm{N}$ is the inverse of the FRF which is defined as .

\subsection{Capacity evaluation for different cellular networks}

The potentially achievable link rates in networks are computed by using the Shannon capacity formula [17] that is given by the following equation 


$$
C=B W \log _{2}(1+C E U s, S I R) \mathrm{bit} / \mathrm{sec}
$$

where $\mathrm{C}$ and $\mathrm{BW}$ denote the channel capacity and frequency BW, respectively.

\section{Results and Discussion}

This work introduces an analytical treatment of different interference mitigation techniques to enhance network QoS. This treatment resulted in closed-form expressions of the worst-cases CEU's SIR and CCU's SIR. These expressions are used to evaluate various performance metrics. Accordingly, a comparison of the considered interference mitigation techniques is presented in this section. This comparison is done through three stages. The first step determines the best scenario in sectored FFR do BF. The second stage defines the superlative scenario in R-A sectored FFR do BF. Then, these scenarios are considered in the last stage of the comparison to represent their respective techniques. Consequently, the outcomes of this work give a clear idea about the behavior of each interference reduction technique. The change of SIR with PL exponent under different BF effects is depicted in Figs. 7-10. From these figures, it is observed, that PL gain increases due to PL exponent increase that in turn compensates the signal attenuation. Consequently, the SIR increases with PL exponent increase. Furthermore, it is noticed, that the performance of sectored FFR do vertical BF scenario is close to that of sectored FFR do 3D BF scenario in low PL exponent range as revealed in Fig. 7. Accordingly, applying vertical BF technique has the preference from the network cost and power consumption points of view. The reason of this result, thanks to the ability of vertical $\mathrm{BF}$ to provide nearly the same SIR values as 3D BF with lower cost and power consumption. However, this scenario lost its superiority in high PL exponent range. This result can be attributed, to the inability of vertical BF technique to direct in a precise way the beam to the desired user when several users have the same elevation angle. Hence, the interference effect 
increases. Accordingly, the CEU's SIR decreases. Furthermore, it is noticed that in general, the scenario of sectored FFR do 3D BF tops the other scenarios. The reason of this result can be accredited, to the capability of 3D BF to serve several users simultaneously even who have the same elevation or azimuth angle. Therefore, the interference sources are reduced. Accordingly, the interfering signals are decreased. Hence, the CEU's SIR increases. Consequently, this scenario attains the highest SIR value in sectored FFR do BF technique. So, this result denotes the first stage in the comparison.

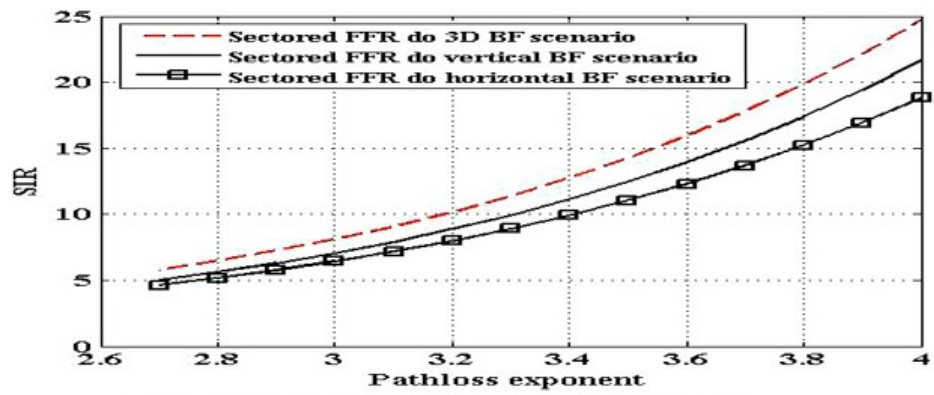

Fig. 7 SIR against PL exponent when sectored FFR do BF is used.

Fig. 7 SIR against PL exponent when sectored FFR do BF is used.

The next results represent the second stage of the comparison. This step defines the best three scenarios in R-A sectored FFR do BF technique. As mentioned above, nine different scenarios are considered in this technique according to the applied BF technique with each configuration. The outcomes of this stage contribute to determining the best scenario to be used with each BF technique. 


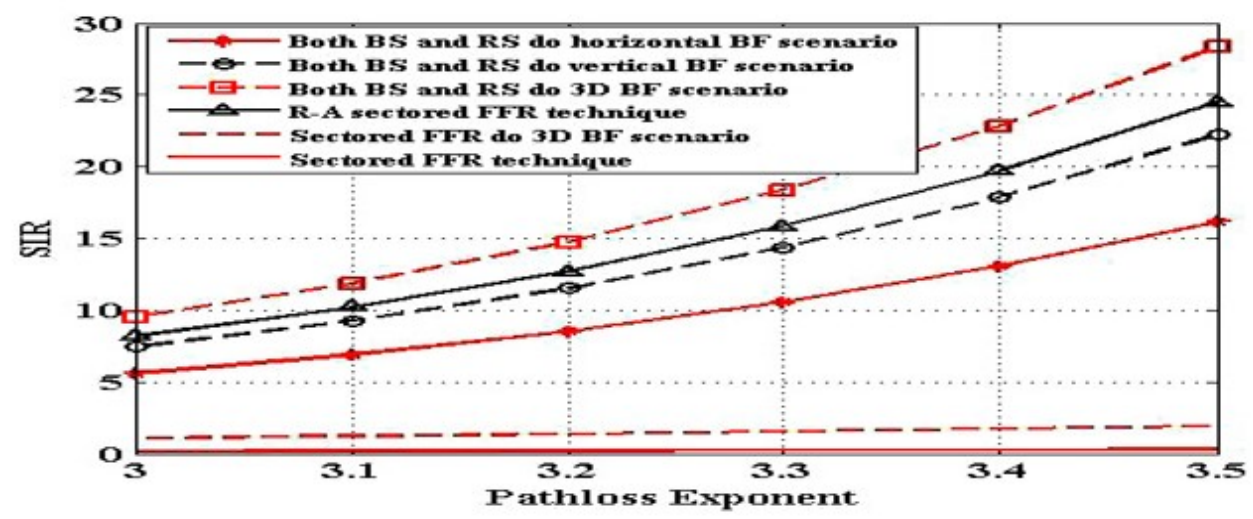

Fig. 8 SIR of different interference mitigation techniques against PL exponent when both RS and BS do various BF techniques.

Fig. 8 depicts the change of SIR with PL exponent when both RS and $\mathrm{BS}$ do different BF techniques. It is observed, that scenario of both BS and $\mathrm{RS}$ do $3 \mathrm{D} \mathrm{BF}$ is superior to other scenarios and techniques. The reason of this result thanks, to the ability of $3 \mathrm{D} \mathrm{BF}$ to direct the signal to desired user without causing interference. Therefore, CEU's SIR increases. Accordingly, applying 3D BF with both BS and RS increases the achieved CEU's SIR of the two time slots. Consequently, this scenario achieves the highest CEU's SIR. Furthermore, it is noticed, R-A sectored FFR outdoes other scenarios of applying BF. This result can be attributed, to the inability of $2 \mathrm{D} B F$ technique to direct the beam in a precise way towards the desired user only. Hence, the interference effect increases especially between CEUs who have the same elevation or azimuth angle. Accordingly, the CEU's SIR decreases. So, applying vertical or horizontal BF with both BS and RS has respectively the third and fourth opportunity. 


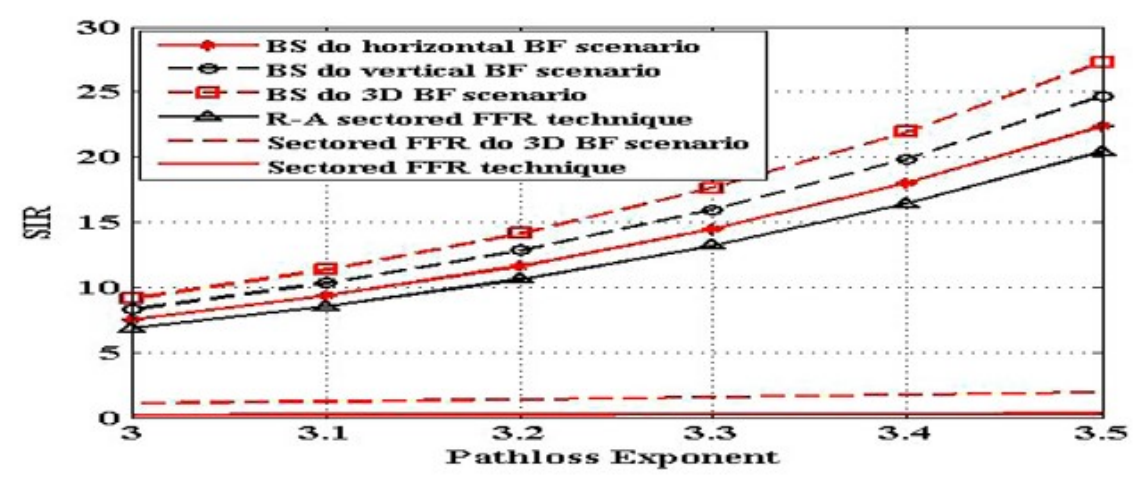

Fig. 9 SIR of various interference mitigation techniques against PL exponent when BS do different BF techniques.

Fig. 9 displays the variation of SIR with PL exponent when BS do different $\mathrm{BF}$ techniques. It is observed, that performance of R-A sectored FFR technique is close to that of the three scenarios of BS do BF in low PL exponent range. Accordingly, this technique has the preference in use from the network budget and energy reservation perspectives. The reason of this result, thanks to the ability of R-A sectored FFR technique to provide nearly the same SIR values as BS do BF with lower cost and power consumption. However, in high $\mathrm{PL}$ exponent range, the scenario of $\mathrm{BS}$ do $3 \mathrm{D} \quad \mathrm{BF}$ is superior to other scenarios and techniques. This result can be credited, to the capability of the antenna array system in 3D BF to trace the user location and supply a dynamic coverage. Therefore, the system performance increases which affects positively the reliability of the communication. Hence, the CEU's SIR increases. Accordingly, applying 3D BF with BS enables the system to achieve high SIR. Consequently, the scenario of BS do 3D BF outperforms other scenarios and techniques. Fig. 10 depicts the change of SIR with PL exponent when the different BF techniques are used with RS. It is mentioned above, that $3 \mathrm{D} \mathrm{BF}$ has the capability to dynamically focus the desired beam to the user of interest only. Therefore, the interference effect decreases. Besides the received signal 
power increases. Consequently, the CEU's SIR increases. Thus, applying 3D BF with RS contributes to achieving much higher CEU's SIR. In addition, it is observed, that horizontal $\mathrm{BF}$ technique can't distinguish many users simultaneously who have the same down-tilt angle. Therefore, more interference is resulted. Additionally, the negative effect of this interference increases as the access link decreases. Thus, applying horizontal or vertical BF with RS increases this effect. Accordingly, the CEU's SIR value decreases. Consequently, R-A sectored FFR technique is preferable in use when horizontal or

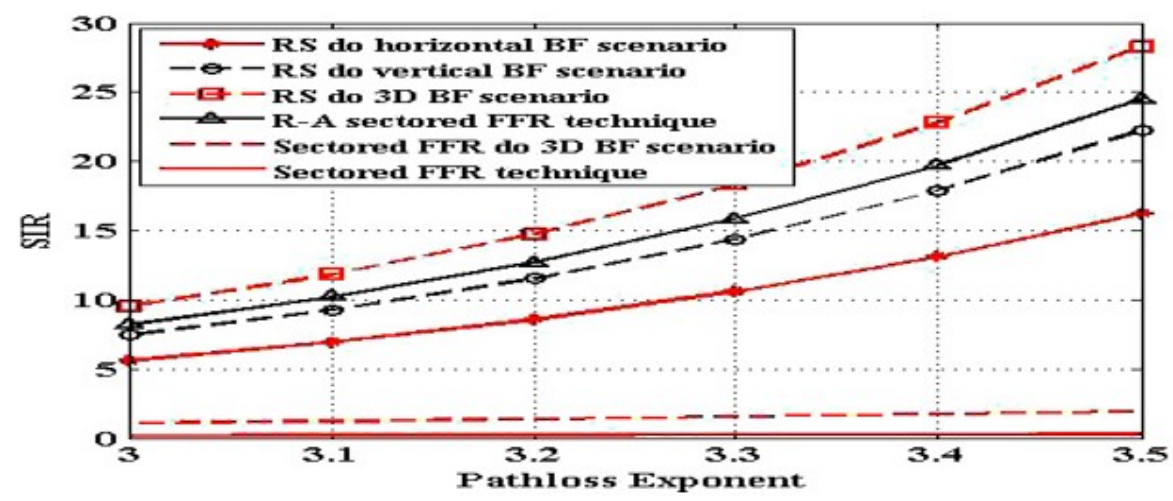

Fig. $10 \mathrm{SIR}$ of different interference mitigation techniques against PL exponent when RS do different BF techniques.

vertical BF is used. Also, it is noted, that the superiority of using this technique appears clearly in the high PL exponent range. These results contribute to saving the power and reducing network cost. Table 5 illustrates the comparison results between the different configurations of R-A sectored FFR do BF according to the applied BF technique. Furthermore, SIR value is the evaluation metric, where the best scenario gives the highest CEU's SIR. For example, when 3D BF technique is applied, the best scenario to be used is the scenario of both BS and RS do BF followed by the scenario of BS do BF only. Additionally, the configuration of RS do BF perf orms worse than other configurations when $3 \mathrm{D} \mathrm{BF}$ technique is used. This 
order provides an accurate and easy way to determine the best configuration to be used with a certain applied BF technique.

Table 5 Comparison of R-A sectored

FFR configurations cooperative with different BF techniques

\begin{tabular}{|c|c|c|c|}
\hline $\begin{array}{c}\text { Performance evaluation } \\
\text { metric }\end{array}$ & 3D BF technique & $\begin{array}{c}\text { Vertical BF } \\
\text { technique }\end{array}$ & $\begin{array}{c}\text { Horizontal BF } \\
\text { technique }\end{array}$ \\
\hline \hline \multirow{2}{*}{ Highest SIR value } & Both BS and RS & BS & BS \\
\cline { 2 - 4 } Lowest SIR value & BS & RS & RS \\
\cline { 2 - 4 } & RS & Both BS and RS & Both BS and RS \\
\hline
\end{tabular}

\section{Conclusion}

In this paper, the network performance improvements by reducing CCI impact is addressed. These improvements are studied through four interference mitigation techniques. These techniques represent traditional sectored FFR, sectored FFR do BF, R-A sectored FFR and RA sectored FFR do BF. Additionally, each one of the techniques which uses BF studies the horizontal, vertical and 3D $\mathrm{BF}$ techniques. Consequently, sectored FFR do BF contains three different scenarios. These scenarios denote sectored FFR do horizontal BF, sectored FFR do vertical BF and sectored FFR do 3D BF. In addition, there are three different considered configurations of applying BF in R-A sectored FFR do BF. These configurations indicate RS do BF only, BS do BF only and both RS and BS do BF. Moreover, each configuration consists of three different scenarios according to the deployed BF technique. Consequently, nine different scenarios are studied. A comparison of all scenarios in different techniques is introduced to enhance the network QoS. Analytical treatments are conducted. These treatments result in closed form expressions for worst case CCU' SIR, CEU's SIR and inner 
radius. Additionally, different performance metrics are evaluated to compare the performance of different interference mitigation techniques. The results show, that as unpredicted, R-A sectored FFR technique outperforms the other techniques in low SIR threshold range and high PL exponent range. Therefore, this technique is preferable in use from SIR and network cost perspectives. Accordingly, there is no need to deploy BF techniques under these conditions. Furthermore, vertical BF shows performance close to 3D BF in low PL exponent range. Therefore, applying vertical BF is preferable with regard to the network budget and energy preserve. Moreover, it is observed, that configuration of BS do BF has the preference in use when horizontal or vertical BF is applied. The reason of this result can be attributed, to the large distance of direct link which contributes to reducing the interference impact. Therefore, the CEU's SIR increases. Accordingly, this configuration is much superior to other configurations when one of these two BF techniques is employed. Furthermore, it is noted, that $3 \mathrm{D}$ BF has the ability to provide a dynamic coverage for the CEU. Thus, interference among users decreases. Consequently, the configuration of both RS and BS do BF outdoes the other configurations when $3 \mathrm{D} \mathrm{BF}$ is deployed. However, as unexpected, it performs worse than them when the other BF techniques are applied. These results provide much higher CEU's SIR enhancements. Thus, the network performance can be improved. Therefore, incoming calls will experience lower blocking probability. Hence, the cell can support a greater traffic density of users. Consequently, the total network cost is reduced.

\section{References}

1. J. Shi, Z. Song and Q. Ni, "Distributed resource allocation assisted by intercell interference mitigation in downlink multicell MC DS-CDMA systems", IEEE Transactions on Wireless Communications, Vol. 16, Pp. 1250-1266, 2017. doi: 10.1109/TWC.2016.2642961

2. O. H. Elgzzar, I. I. Mahmoud, S. Hashima and H. A. Konber, "Analysis of downlink sectored frequency reuse cellular systems combined with different 
beamforming techniques", $201611^{\text {th }}$ International Conference on Computer Engineering and Systems (ICCES), Pp. 401-406, 2016. doi: http://doi.org/10.1109/ICCES.2016.7822038.

3. J. G. Morales, G. Femenias and F. R. Palou, "Analysis and optimization of FFR-aided OFDMA-based heterogeneous cellular networks", IEEE Access, Vol. 4, Pp. 5111-5127, 2016. doi: 10.1109/ACCESS.2016.2599026

4. F. Chiti, R. Fantacci and L. Pierucci, "Dynamic multicast link selections for buffer-aided relaying networks", International Journal of Communication System, Vol. 29, Pp. 1790-1804, 2016. doi: 10.1002/dac.3145

5. E. M. Taghavi and G. A. Hodtani, "Energy-efficient communications over multiple access relay channels", International Journal of Communication System, Vol. 30, Pp. 1 - 13, 2017. doi: https://doi.org/10.1002/dac.3368

6. S. I. Chu, "Secrecy performance analysis of AF relaying with relay selection scheme over Nakagami-m fading channels", International Journal of Communication System, Vol. 31, Pp. 1- 26, 2018. doi: https://doi.org/10.1002/dac.3738

7. K. Chaitanya and P. Muthuchidambaranathan, "Performance analysis of decode and forward relaying over dual-hop fading", International Journal of Electronics and Communications, Vol. 73, Pp. 84-88, 2016. doi: http://dx.doi.org/10.1016/j.aeue.2016.12.026

8. F. E. Airod, H. Chafnaji and A. Tamtaoui, " A comparative study of full-duplex relaying schemes for low latency applications", International Journal of Communication System, Vol. 31, Pp. 1 - 13, 2018. doi: https://doi.org/10.1002/dac.3728

9. W. Chen,"CAO-SIR: channel aware ordered successive relaying", IEEE Transactions on Wireless Communications, Vol. 13, Pp. 6513-6527, 2014. doi: 10.1109/TWC.2014.2363453

10. S. Teodoro, A. Silva, J. M. Gil and A. Gameiro, "Novel precoded relay-assisted algorithm for cellular systems", EURASIP Journal on Wireless Communications and Networking, Vol. 2010, Pp. 1-10, 2010. doi: https://doi.org/10.1155/2010/414657 
11. M. I. Khalil, S. M. Berber and K. W. Sowerby, "High SNR approximation for performance analysis of two-way multiple relay networks", Physical Communication, Vol. 24, Pp. 62-70, 2017. doi: http://dx.doi.org/10.1016/j.phycom.2017.04.007

12. W. Hou, M. Jiang and S. Feng, "Beamforming for inter-relay interference reduction in MIMO-aided two-path successive relaying", Wireless Communications and Mobile Computing, Vol. 16, Pp. 2274-2286, 2016. doi: $10.1002 / \mathrm{wcm} .2682$

13. O. Taghizadeh, J. Zhang and M. Haardt, "Transmit beamforming aided amplifyand-forward MIMO full-duplex relaying with limited dynamic Range", Signal Processing, Vol. 127, Pp. 266-281， 2016 . doi: http://dx.doi.org/10.1016/ j.sigpro.2016.02.026

14. A. R. Kebrya, B. Liang and M. Dong, "Interference minimization in cooperative relay beamforming with multiple communicating pairs", IEEE Transactions on Wireless Communications, Vol. 16, Pp.6514- 6527, 2017. doi: 10.1109/TWC.2017.2724508

15. D. Bilios, C. Bouras, V. Kokkinos, A. Papazois and G. Tseliou, "Selecting the optimal fractional frequency reuse scheme in long term evolution networks", Wireless Personal Communications, Vol. 71, Pp. 26932712, 2013. doi: http://doi.org/10.1007/s11277-012-0965-z.

16. I. I. Mahmoud, O. H. Elgzzar, S. Hashima and H. A. Konber, "An accurate model of worst case signal to interference ratio for frequency reuse cellular systems", $201611^{\text {th }}$ International Conference on Computer Engineering and Systems (ICCES), Pp. 393-400, 2016. doi: http://doi.org/10.1109/ICCES.2016.7822037.

17. L. C. Wang and C. J. Yeh, "3-cell network MIMO architectures with sectorization and fractional frequency reuse", IEEE Journal on Selected Areas in Communications, Vol. 29, Pp. 1185-1199, 2011. doi: http://doi.org/10.1109/JSAC.2011.110607.

18. Y. Li, X. Ji, D. Liang and Y. Li, "Dynamic beamforming for threedimensional MIMO technique in LTE-advanced networks", International 
Journal of Antennas and Propagation, Vol. 2013, Pp.1-8, 2013. doi: http://doi.org/10.1155/2013/764507.

19. A. Khelil, L. Talbi, D. Slimani and J. Lebel, "MRC SC-FDMA scheme performance evaluation based on measurements at $30 \mathrm{GHz}$ for $5 \mathrm{G}$ communications", Physical Communication, Vol. 25, Pp. 462-468, 2017. doi: http://dx.doi.org/10.1016/j.phycom.2017.08.018

20. L. Han and W. Zou, "Performance analysis of relay-assisted uplink massive MIMO systems with zero-forcing receiver", Physical Communication, Vol. 25, Pp. 304-309, 2017. doi: http://doi.org/10.1016/j.phycom.2017.02.001.

21. H. Q. Ngo and E. G. Larsson, "Linear multihop amplify-and-forward relay channels: error exponent and optimal number of hops", IEEE Transactions on Wireless Communications, Vol. 10, Pp. 3834-3842, 2011. doi: http://doi.org/10.1109/TWC.2011.092011.102194.

22. J. M. Kelif, M. Coupechoux and P. Godlewski, "On the dimensioning of cellular OFDMA networks", Physical Communication, Vol. 5, Pp.10-21, 2012. doi: https://doi.org/10.1016/j.phycom.2011.09.008.

23. D. B. Cheikh, J-M. Kelif, M. Coupechoux and P. Godlewski, "SIR distribution analysis in cellular networks considering the joint impact of path-loss, shadowing and fast fading", EURASIP Journal on Wireless Communications and Networking, Vol. 2011, Pp.1-10, 2011. doi: 10.1186/1687-1499-2011-137.

24. H. Zhang, X. Wang, Y. Liu, L. Zheng and T. M. Bohnert, "Resource allocation for relay-assisted OFDMA systems using inter-cell interference coordination", EURASIP Journal on Wireless Communications and Networking, Vol. 2012, Pp.1-10, 2012. doi: 10.1186/1687-1499-2012-156. 